Doug Geisler, Eva K. Grebel, and Dante Minniti, eds.

\title{
Globular Cluster Specific Frequencies in Coma
}

\author{
A. Marín-Franch
}

Instituto de Astrofísica de Canarias, Vía Láctea s/n, 38200 La Laguna, Tenerife, Spain

\section{A. Aparicio}

Instituto de Astrofísica de Canarias, Vía Láctea s/n, 38200 La Laguna, Tenerife, Spain

\begin{abstract}
.
Globular cluster systems (GCSs) have been observed in 17 elliptical galaxies located in Coma. Surface brightness fluctuations (SBF) and a 2.5-m ground-based telescope (INT, La Palma) have been used to determine total populations of Globular clusters (GCs) and specific frequency $\left(S_{N}\right)$ has been evaluated for each individual galaxy.
\end{abstract}

\section{Introduction}

The SBF technique was introduced by Tonry \& Schneider (1988) with the aim of measuring distances. Accurate estimates of distances can be obtained up to $\sim 40 \mathrm{Mpc}$. In the case of more distant galaxies, SBF can be used to determine GCs populations (Blakeslee \& Tonry 1995). In this work, the SBF technique is used to evaluate the GCS properties of elliptical galaxies in the Coma cluster. Coma is a good laboratory for studying GCSs, because it is a rich cluster and its ellipticals have a wide range of luminosities and environmental densities.

\section{Results}

In table 1 a summary of results is given. For each galaxy, the value of $M_{V}^{T O T}$, taken from the RC3 catalogue and adopting a distance modulus for Coma of 34.71 (van den Bergh 1992); the distance to the central galaxy NGC 4874 $\left(R_{N G C 4874}\right)$; the number of GCs obtained for each galaxy $\left(N_{G C}^{t o t}\right)$ and the result for $S_{N}$ are shown.

These results reveal enormous differences in $S_{N}$ which varies across an order of magnitude from galaxy to galaxy. Extreme cases are the following: a) at the lower end of the range, NGC 4673 has $S_{N}=1.3 \pm 0.4$, a surprising value for an elliptical galaxy, but typical for spiral and irregular galaxies; b) at the upper extreme, MCG $+5-31-063$ has $S_{N}=16.0 \pm 4.2$ and is more likely to belong to a supergiant $\mathrm{cD}$ galaxy than to a "normal" elliptical galaxy. Furthermore, NGC 4874 , the central supergiant cD galaxy of the Coma cluster, exhibits a relatively 
high specific frequency $\left(S_{N}=11.2 \pm 2.8\right)$. The other galaxies studied have $S_{N}$ in the range [3, 9], the mean value being $S_{N}=6.0$.

In order to study possible relations between $S_{N}$ and environment, we plot $S_{N}$ versus the distance $R$ to the central Coma galaxy, NGC 4874 (Fig. 1, left panel). In this plot we do not find any relation between $S_{N}$ and the environmental density for the 17 galaxies. On the other hand, in the right panel of Fig. 1, we plot $S_{N}$ versus $M_{V}^{T O T}$ of each galaxy. Again, no relation between $S_{N}$ and $M_{V}^{T O T}$ is found. The figure is completely dominated by the dispersion between points.

Table 1. Summary of results.

\begin{tabular}{ccccc}
\hline \hline Galaxy & $M_{V}^{T O T}$ & $R_{\mathrm{NGC} 4874}\left({ }^{\prime}\right)$ & $N_{G C}^{t o t}$ & $S_{\mathrm{N}}$ \\
\hline & & & & \\
NGC4874 & $-23.03 \pm 0.19$ & 0 & $18200 \pm 3200$ & $11.2 \pm 2.8$ \\
NGC4889 & $-23.22 \pm 0.19$ & 8.05 & $9600 \pm 1900$ & $4.9 \pm 1.3$ \\
NGC4886 & $-20.85 \pm 0.19$ & 7.43 & $1900 \pm 300$ & $8.7 \pm 2.0$ \\
IC4012 & $-19.77 \pm 0.19$ & 10.68 & $600 \pm 300$ & $7.6 \pm 3.9$ \\
IC4021 & $-19.86 \pm 0.19$ & 10.88 & $150 \pm 100$ & $1.8 \pm 1.2$ \\
IC4026 & $-20.12 \pm 0.22$ & 12.62 & $650 \pm 130$ & $5.8 \pm 1.7$ \\
IC4041 & $-20.36 \pm 0.26$ & 16.38 & $470 \pm 90$ & $3.4 \pm 1.0$ \\
IC4045 & $-21.77 \pm 0.19$ & 19.85 & $1090 \pm 360$ & $5.4 \pm 1.0$ \\
IC4051 & $-21.51 \pm 0.22$ & 19.95 & $6250 \pm 870$ & $15.6 \pm 3.8$ \\
IC3976 & $-20.01 \pm 0.22$ & 6.78 & $920 \pm 210$ & $9.1 \pm 2.8$ \\
IC3959 & $-20.72 \pm 0.22$ & 12.67 & $880 \pm 230$ & $4.5 \pm 1.5$ \\
MGC +5 -31 -063 & $-20.32 \pm 0.22$ & 8.93 & $2150 \pm 360$ & $16.0 \pm 4.2$ \\
NGC4839 & $-22.65 \pm 0.19$ & 43.12 & $9900 \pm 1900$ & $8.7 \pm 2.2$ \\
NGC4840 & $-21.01 \pm 0.21$ & 37.43 & $1290 \pm 440$ & $5.1 \pm 2.0$ \\
NGC4816 & $-21.87 \pm 0.26$ & 52.60 & $6200 \pm 1200$ & $11.0 \pm 3.0$ \\
NGC4673 & $-21.91 \pm 0.24$ & 217.07 & $750 \pm 200$ & $1.3 \pm 0.4$ \\
IC3651 & $-21.45 \pm 0.22$ & 290.32 & $2700 \pm 540$ & $7.1 \pm 2.0$ \\
\hline & & & &
\end{tabular}

Formation scenarios for giant ellipticals tend to fall into three basic classes of models: a) in situ models, in which the galaxy condenses by dissipative collapse of gas clouds in one or more major bursts, b) mergers of gas-rich systems, probably disk-type galaxies, and c) accretion of smaller satellites. Various combinations of these extremes are also possible.

In situ models predict a correlation between galaxy properties and its GCS, and this sort of relation is not observed in this study. On the other hand, if a range of possible GC formation efficiencies is allowed, the merger model can account for the $S_{N}$ range observed for elliptical galaxies. If the merger model is to explain the high $-S_{N}$ phenomenom, GC formation must be more efficient in the high $-S_{N}$ galaxies.

No single scenario seems to account for the observed $S_{N}$, so the history of each galaxy must be deduced individualy combining properly the basic models (mergers, in situ and accretions). It is necesary for this purpose to extend the obervational information of each galaxy. Besides the specific frequency and 

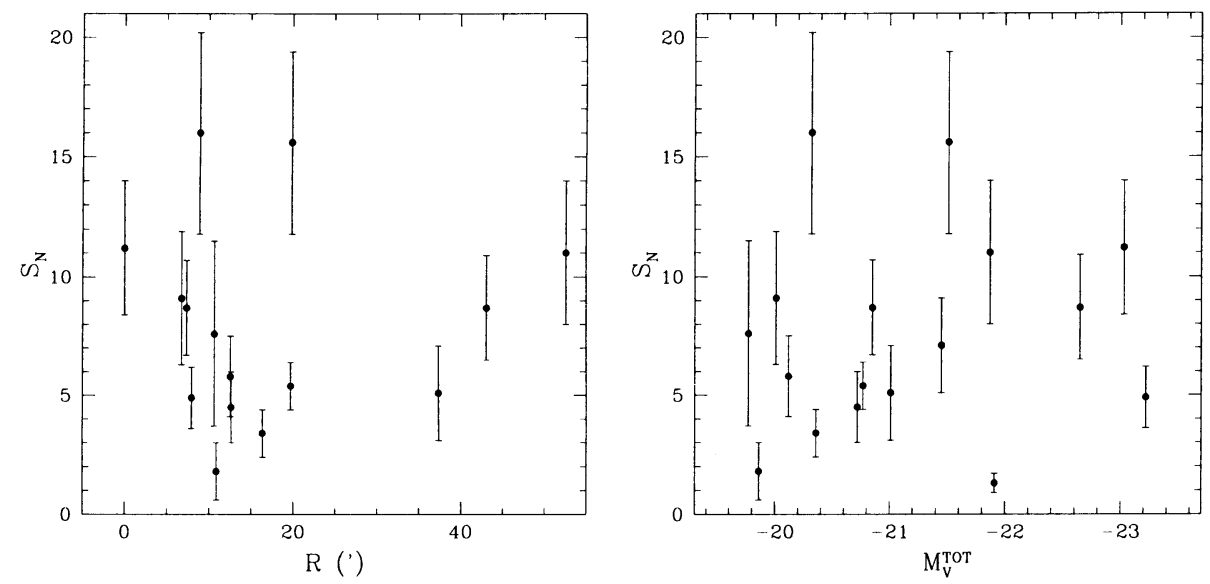

Figure 1. Left panel: $S_{N}$ versus the distance of the host galaxy to the Coma cluster center. Right panel: $S_{N}$ versus $M_{V}^{T O T}$ of the host galaxy.

the radial distribution of the GCS, it is necessary to know the details of the subpopulations (when they exist) and the kinematics of the GCs. In this way, detailed HST observations of the extreme cases NGC 4673 and MCG +5 -31 -063 could be a good starting point, because these "rare" galaxies can show characteristics found nowhere else, thus providing valuable information in testing the different scenarios.

\section{References}

Blakeslee, J. P., \& Tonry, J. L. 1995, ApJ, 442, 579

Tonry, J. L., \& Schneider, D. P. 1988, AJ, 96, 807 